\title{
Review: cognitive behaviour therapy facilitates recovery from depressive disorders in children and adolescents
}

\author{
Harrington $R$, Whittaker J, Shoebridge P, et al. Systematic review of efficacy of cognitive behaviour therapies in childhood and \\ adolescent depressive disorder. BMJ 1998 May 23;316:1559-63.
}

\section{Question}

In children and adolescents with a depressive disorder, is cognitive behaviour therapy (CBT) effective?

\section{Data sources}

Studies were identified by searching Medline (1966-97), PsycLit, and the Cochrane Library; by manually searching all journals that publish randomised controlled trials (RCTs) in the field; by reviewing conference proceedings and the reference lists from reviews and book chapters; and by contacting authors and investigators working in this specialty.

\section{Study selection}

Studies were selected if they were RCTs that investigated the effectiveness of CBT in young people between the age of 6 and 18 years with depressive disorders diagnosed using standardised criteria. Studies of family therapy or interpersonal therapy were excluded.

\section{Data extraction}

Data were extracted on patient characteristics, type of CBT, type of comparative condition, and rate of remission from depressive disorder. The quality of each study was also rated by the authors using a set of predefined dimensions.

\section{Main results}

6 trials were selected involving young people between 8 and 19 years of age that compared CBT with a heterogenous set of mostly inactive interventions (no comparisons with medication were found). The control interventions were primarily either inactive (eg, waiting list) or attention placebo conditions (eg, relaxation training, art exercises). A metaanalysis was done showing that there was no significant heterogeneity across the trials. An "end of treatment" pooled analysis showed that more patients assigned to CBT had a remission of their depressive disorder than did those assigned to a control group $\{p<0.001\}$ (table). An intention to treat analysis did not substantially change the results. The studies included mostly mild to moderate cases of depression and were of only moderate quality.

\section{Conclusion}

Cognitive behaviour therapy facilitates recovery from depressive disorders in children and adolescents.

* p value calculated from data in article.

Cognitive behaviour therapy $(C B T) v$ an inactive control intervention in young people with depressive disorderst

\begin{tabular}{lllll}
\hline & \multicolumn{2}{l}{ Weighted event rates } & \\
\cline { 2 - 3 } Outcome & $C B T$ & Control & RBI $(95 \%$ CI $)$ & NNT (CI) \\
\hline $\begin{array}{l}\text { Remission of } \\
\text { depressive disorder }\end{array}$ & $64 \%$ & $36 \%$ & $73 \%(38$ to 116$)$ & 4 (3 to 6) \\
\hline
\end{tabular}

†Abbreviations defined in glossary; RBI, NNT, and CI calculated from data in article.

Sources of funding: McArthur Foundation Research Network on Psychopathology and Development and NHS Executive North West and Department of Health

For correspondence: Professor R Harrington, Department of Child and Adolescent Psychiatry, Royal Manchester Children's Hospital, Manchester M27 1HA, UK. Fax +44 (0)161 7282294.

\section{Commentary}

This review by Harrington et al confirms that CBT, which generally uses a focused, active, problem solving oriented, and time limited approach, is effective with younger clinically depressed patients. The results also echo a long documented finding in depression treatment research across the age span-namely, that there is a high placebo response rate, which can exceed $50 \%$. This may mean that there are therapeutic (although not well defined) elements in "attention placebo" interventions that may derive from the relationship of the professional to the depressed patient; or it may point to the time limited course of the disorder.

The children and adolescents treated in the studies reviewed by Harrington $e t$ al had to meet specific psychiatric diagnostic criteria for depressive disorder. Thus, these young people were symptomatic and impaired, although possibly no more than about $25 \%$ of them had severe forms of depression. The results therefore indi- cate that the majority of children and adolescents with depressive disorder in ambulatory settings are responsive to focused non-pharmacological treatments targeted at their depression. This is important information because there is not yet a compelling body of data on the efficacy of pharmacotherapy for depression in children and adolescents.

Harrington et al also suggest that CBT may be more effective in young people with mild to moderate depression than in those whose depression is severe. Similar trends have been detected in studies of depressed adults. Just as with most psychologically based therapies, CBT requires active engagement on the part of the patient, including some willingness and motivation to implement changes in thinking and behaviour. Because severely depressed individuals often are unable to motivate themselves and may be psychomotorically retarded, it is not surprising that they are less likely to respond to CBT.
Although it is now well documented that depressive disorders in young people represent a disabling and recurrent condition which requires professional attention, there is a striking paucity of treatment outcome studies. Additionally, existing treatments typically involve a "downward" extension of some therapeutic modality that had been designed for depressed adults, but without much attention being paid to developmental issues in their application. More effective treatments for depressed young people therefore may require accommodating their less mature cognitive, emotional, and behavioural repertoires, their substantial reliance on their parents and other adults, and consideration of their developing physiological status.

Maria Kovacs, $\mathrm{PhD}$

University of Pittsburgh School of Medicine Pittsburgh, Pennsyluania, USA 